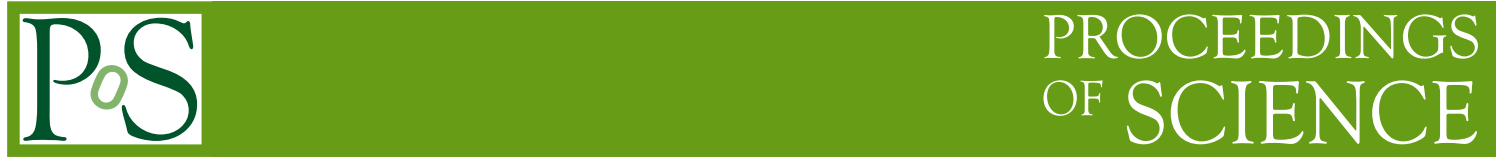

\title{
Two-loop corrections to the Lamb shift
}

\section{Matthew Dowling, Jorge Mondéjar, Jan H. Piclum*and Andrzej Czarnecki}

University of Alberta, Department of Physics, Edmonton, Alberta, Canada T6G 267

E-mail: mdowling@phys.ualberta.ca, jmonde@phys.ualberta.ca,

jpiclum@phys.ualberta.ca, andrzej.czarnecki@ualberta.ca

We present a new calculation of the order $\alpha^{2}(Z \alpha)^{5}$ correction to the Lamb shift, which uses methods developed for multi-loop calculations.

RADCOR 2009 - 9th International Symposium on Radiative Corrections (Applications of Quantum Field Theory to Phenomenology)

October 25-30 2009

Ascona, Switzerland

\footnotetext{
${ }^{*}$ Speaker.
} 


\section{Introduction}

The Lamb shift in hydrogen was discovered in 1947 [1]. Since then, developments in spectroscopy have led to very precise experimental values for the $1 S$ Lamb shift $[2,3,4,5,6]$, making it the best test of Quantum Electrodynamics for an atom. On the theory side, much effort has been put into the calculation of higher order corrections to match the experimental precision (cf. Ref. [7] for a review of the theory of light hydrogen-like atoms).

In the perturbative calculation it is important to correctly account for the presence of three different small parameters. $Z \alpha$ describes the binding effects of an electron to a nucleus with charge number $Z$. Self-interactions of the electron lead to additional powers of $\alpha$, but not of $Z$. Therefore, it is useful to keep a generic $Z$ to distinguish the two different effects, even though our main application is hydrogen where $Z$ is equal to one. The third small parameter is the ratio of electron and nucleus masses, $m / M$. The Lamb shift is of order $\alpha(Z \alpha)^{4}$. Corrections also include logarithms of $Z \alpha$ and $m / M$. At present, all second-order corrections in $\alpha$ and $Z \alpha$ are known, as well as some third order ones $[8,9]$.

Another important correction is due to the spatial distribution of the nuclear charge. At the moment, the theoretical prediction for hydrogen is limited by the uncertainty in the measurement of the proton root mean square charge radius. However, new measurements $[10,11]$ of the Lamb shift in muonic hydrogen are expected to improve the knowledge of this parameter, soon. The advantage of muonic hydrogen is the greater sensitivity to the proton charge distribution due to the larger mass, and thus stronger binding, of the muon.

Here we present a new calculation of the second-order non-recoil corrections of order $\alpha^{2}(Z \alpha)^{5}$. The full result for these corrections was presented first in Ref. [12] and improved in Refs. [13, 14]. Our result [15] is compatible with the previous ones and has better precision.

\section{Calculation}

Since we are only interested in non-recoil corrections, we consider the scattering of an onshell electron with an on-shell nucleus, where both external momenta have vanishing space-like components. Furthermore, we only have to calculate the leading term in the expansion around $M \rightarrow \infty$. In this particular case we can simplify the interaction with the nucleus when we consider the diagram with direct photon exchange together with the diagram with crossed photon exchange, as depicted in Fig. 1. In the infinite mass limit, the nucleus propagators become static propagators. However, due to the different momentum flow in the two diagrams, the sum of the two becomes the difference of two terms which differ only in the sign of the $i \varepsilon$ prescription of these propagators.

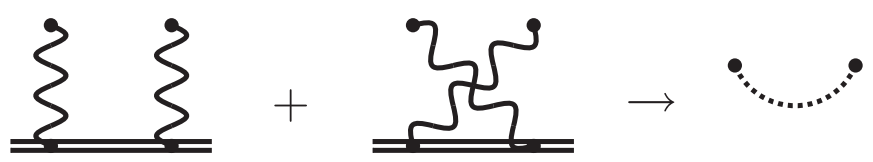

Figure 1: The effective propagator for the interaction with the nucleus. Wavy and double lines denote photons and the nucleus, respectively. 


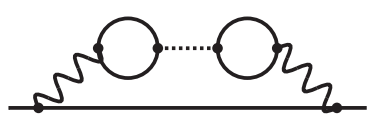

$(a)$

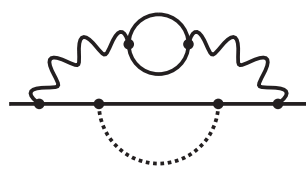

(b)

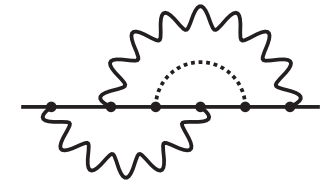

(c)

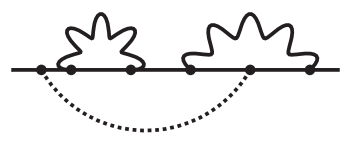

$(d)$

Figure 2: Sample diagrams. Solid, wavy, and dotted lines denote electrons, photons, and the effective propagator, respectively.

Neglecting the Dirac structure, we have

$$
\frac{1}{(N+k)^{2}-M^{2}+i \varepsilon}+\frac{1}{(N-k)^{2}-M^{2}+i \varepsilon} \stackrel{M \rightarrow \infty}{\rightarrow} \frac{1}{2 N \cdot k+i \varepsilon}-\frac{1}{2 N \cdot k-i \varepsilon}=\frac{i \pi}{M} \delta\left(k^{0}\right),
$$

where $N=(M, \overrightarrow{0})$ and $k$ are the nucleus and photon momentum, respectively. The latter is considered to scale like the electron mass, which is much smaller than the nucleus mass. Thus, we have to compute three-loop electron self-energy diagrams with two photons and one effective propagator, which is defined by Fig 1. Sample diagrams are depicted in Fig. 2.

Our calculation proceeds as follows. We use QGRAF [16] to generate the Feynman diagrams, and $q 2 e$ and $\exp [17,18]$ to turn them into FORM [19] readable code. Finally, we use MATAD 3 [20] to do the Dirac algebra and express all diagrams in terms of scalar integrals, using self-made routines. The next step is the reduction to so-called master integrals using integration-by-parts identities [21, 22]. For this we use the program FIRE [25], which is a Mathemat ica implementation of the so-called Laporta algorithm [23, 24].

Sample master integrals are depicted in Fig. 3. We used different methods for their evaluation. Simpler integrals like the one in Fig. 3(a) were computed with the Mellin-Barnes method [26, 27], using the Mathematica packages MB [28] and MBresolve [29]. For more complicated integrals, we used sector decomposition [30,31], as implemented in the program FIESTA [32, 33]. The most complicated master integral is the one in Fig. 3(c). Fortunately, this integral is finite, and we were able to derive a Feynman-parameter representation which could be integrated with the help of the Cuba library [34]. Results for all master integrals are given in Ref. [15].

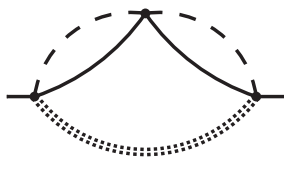

$(a)$

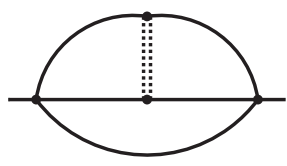

(b)

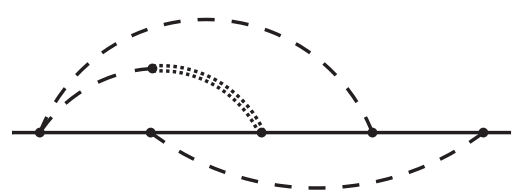

(c)

Figure 3: Sample master integrals. Solid and dashed lines denote scalar massive and massless propagators, respectively. Dotted double lines denote the delta function.

\section{Results}

In order to present our final result, we split the Feynman diagrams into two subsets: diagrams with closed electron loops (cf. Fig. 2(a) and $(b)$ ) are denoted by the subscript $v p$, and diagrams 
without closed electron loops (cf. Fig. $2(c)$ and $(d)$ ) are denoted by the subscript $n v p$. Our results for the corresponding energy shifts are

$$
\begin{aligned}
\delta E_{v p} & =\frac{\alpha^{2}(Z \alpha)^{5}}{\pi n^{3}}\left(\frac{\mu}{m}\right)^{3} m[0.86281422(3)], \\
\delta E_{n v p} & =\frac{\alpha^{2}(Z \alpha)^{5}}{\pi n^{3}}\left(\frac{\mu}{m}\right)^{3} m[-7.72381(4)],
\end{aligned}
$$

where $\mu=m M /(m+M)$ denotes the reduced mass of the atom, and $n$ is the principal quantum number. Results for individual diagrams can be found in Ref. [15].

The best results for the two subsets prior to our calculation have been published in Ref. [35] (cf. Ref. [7] for references of partial results) and Ref. [13], respectively. Our results agree with the previous ones within the error bars. However, we improve the precision by two orders of magnitude for $\delta E_{v p}$, and a little over one order of magnitude for $\delta E_{n v p}$.

The total result reads

$$
\delta E=\frac{\alpha^{2}(Z \alpha)^{5}}{\pi n^{3}}\left(\frac{\mu}{m}\right)^{3} m[-6.86100(4)]
$$

and the corresponding energy shifts for the $1 S$ and the $2 S$ states in hydrogen are

$$
\begin{aligned}
& \delta E_{1 S}=-296.866(2) \mathrm{kHz}, \\
& \delta E_{2 S}=-37.1082(3) \mathrm{kHz} .
\end{aligned}
$$

\section{Acknowledgments}

We are grateful to A.V. and V.A. Smirnov for providing us with a beta version of FIESTA 2 prior to publication. JHP thanks the organisers of "RADCOR 2009" for an interesting conference. This work was supported by the Natural Sciences and Engineering Research Council of Canada and the Alberta Ingenuity Foundation. The Feynman diagrams were drawn using Axodraw [36] and Jaxodraw 2 [37].

\section{References}

[1] W. E. Lamb and R. C. Retherford, Phys. Rev. 72 (1947) 241.

[2] D. J. Berkeland, E. A. Hinds and M. G. Boshier, Phys. Rev. Lett. 75 (1995) 2470.

[3] M. Weitz et al., Phys. Rev. A 52 (1995) 2664.

[4] S. Bourzeix et al., Phys. Rev. Lett. 76 (1996) 384.

[5] T. Udem, A. Huber, B. Gross, J. Reichert, M. Prevedelli, M. Weitz and T. W. Hänsch, Phys. Rev. Lett. 79 (1997) 2646.

[6] C. Schwob et al., Phys. Rev. Lett. 82 (1999) 4960.

[7] M. I. Eides, H. Grotch and V. A. Shelyuto, Phys. Rept. 342 (2001) 63 [arXiv:hep-ph/0002158].

[8] K. Pachucki, Phys. Rev. A 63 (2001) 042503.

[9] M. I. Eides and V. A. Shelyuto, Can. J. Phys. 85 (2007) 509 [arXiv:physics/0612244]. 
[10] A. Antognini et al., AIP Conf. Proc. 796 (2005) 253.

[11] B. Lauss, Nucl. Phys. A 827 (2009) 401C [arXiv:0902.3231 [nucl-ex]].

[12] K. Pachucki, Phys. Rev. Lett. 72 (1994) 3154.

[13] M. I. Eides and V. A. Shelyuto, Phys. Rev. A 52 (1995) 954 [arXiv:hep-ph/9501303].

[14] M. I. Eides and V. A. Shelyuto, Pisma Zh. Eksp. Teor. Fiz. 61 (1995) 465 [JETP Lett. 61 (1995) 478$].$

[15] M. Dowling, J. Mondéjar, J. H. Piclum and A. Czarnecki, arXiv:0911.4078 [hep-ph].

[16] P. Nogueira, J. Comput. Phys. 105 (1993) 279.

[17] R. Harlander, T. Seidensticker and M. Steinhauser, Phys. Lett. B 426 (1998) 125 [arXiv:hep-ph/9712228].

[18] T. Seidensticker, arXiv:hep-ph/9905298.

[19] J. A. M. Vermaseren, arXiv:math-ph/0010025.

[20] M. Steinhauser, Comput. Phys. Commun. 134 (2001) 335 [arXiv:hep-ph/0009029]; URL: http://www-ttp.particle.uni-karlsruhe.de/ ms/software.html.

[21] F. V. Tkachov, Phys. Lett. B 100 (1981) 65.

[22] K. G. Chetyrkin and F. V. Tkachov, Nucl. Phys. B 192 (1981) 159.

[23] S. Laporta and E. Remiddi, Phys. Lett. B 379 (1996) 283 [arXiv:hep-ph/9602417].

[24] S. Laporta, Int. J. Mod. Phys. A 15 (2000) 5087 [arXiv:hep-ph/0102033].

[25] A. V. Smirnov, JHEP 0810 (2008) 107 [arXiv:0807.3243 [hep-ph]].

[26] V. A. Smirnov, Phys. Lett. B 460 (1999) 397 [arXiv:hep-ph/9905323].

[27] J. B. Tausk, Phys. Lett. B 469 (1999) 225 [arXiv:hep-ph/9909506].

[28] M. Czakon, Comput. Phys. Commun. 175 (2006) 559 [arXiv:hep-ph/0511200].

[29] A. V. Smirnov and V. A. Smirnov, Eur. Phys. J. C 62 (2009) 445 [arXiv:0901.0386 [hep-ph]].

[30] T. Binoth and G. Heinrich, Nucl. Phys. B 585 (2000) 741 [arXiv:hep-ph/0004013].

[31] T. Binoth and G. Heinrich, Nucl. Phys. B 680 (2004) 375 [arXiv:hep-ph/0305234].

[32] A. V. Smirnov and M. N. Tentyukov, Comput. Phys. Commun. 180 (2009) 735 [arXiv:0807.4129 [hep-ph]].

[33] A. V. Smirnov, V. A. Smirnov and M. Tentyukov, arXiv:0912.0158 [hep-ph].

[34] T. Hahn, Comput. Phys. Commun. 168 (2005) 78 [arXiv:hep-ph/0404043].

[35] K. Pachucki, Phys. Rev. A 48 (1993) 2609.

[36] J. A. M. Vermaseren, Comput. Phys. Commun. 83 (1994) 45.

[37] D. Binosi, J. Collins, C. Kaufhold and L. Theussl, Comput. Phys. Commun. 180, 1709 (2009) [arXiv:0811.4113 [hep-ph]]. 